\title{
Genetic Algorithm for the Cargo Shunting Cooperation between Two Hub-and-Spoke Logistics Networks
}

\author{
Jingjing Hu iD, Youfang Huang iD \\ Shanghai Maritime University (China) \\ 1498013264@qq.com,ybuang@shmtu.edu.cn
}

Received: January 2019

Accepted: April 2019

\begin{abstract}
:
Purpose: The overstocked goods flow in the hub of hub-and-spoke logistics network should be disposed of in time, to reduce delay loss and improve the utilization rate of logistics network resources. The problem we need to solve is to let logistics network cooperate by sharing network resources to shunt goods from one hub-and-spoke network to another hub-and-spoke network.
\end{abstract}

Design/methodology/approach: The paper proposes the cargo shunting cooperation between two hub-and-spoke networks. Firstly, a hybrid integer-programming model was established to describe the problem, and then a multi-layer genetic algorithm was designed to solve it and two hub-and-spoke networks are expressed by different gene segments encoded by genes. Data of two third-party logistics companies in southern and northern China are used for example analysis, the sensitivity of parameters controlling the flow in the network and the transportation cost between the two networks is analyzed at the last step.

Findings: The hub-and-spoke networks of the two companies in the same area are constructed simultaneously; each hub point that needs to be shunt finds a cooperative hub point in another network. The transfer cost coefficient between two networks and the volume of cargo flow in the network can affect the computation of hubs that needed to be shunt and the corresponding cooperation hubs in the other network.

Originality/value: Previous researches on hub-and-spoke logistics network focus on one logistics network, while we study the cooperation and interaction between two hub-and-spoke networks. It shows that two hub-and-spoke network can cooperate across the network to shunt the goods in the hub and improve the operation efficiency of the logistics enterprise.

Keywords: hub-and-spoke network cooperation, shunt goods, multi-layer genetic algorithm, third-party logistics company

\section{To cite this article:}

Hu, J., \& Huang, Y. (2019). Genetic algorithm for the cargo shunting cooperation between two hub-and-spoke logistics networks. Journal of Industrial Engineering and Management, 12(2), 356-372. https://doi.org/10.3926/jiem.2815 


\section{Introduction}

Third Party Logistics (TPL) Company has its own mature logistics network, which is a resource that can be leveraged and integrated. Due to the increasing cargo flow pressures in the hubs or links of the logistics network in high season, for a TPL company, the increased cargo flow in a period of time will far exceed its handling capacity, but TPL companies can hardly build a new logistics facility in a short time. These goods beyond capacity still need to be disposed of in time, then the logistics network cooperation between different TPL companies may be considered.

For the research of hub-and-spoke logistics network, some research take in account the various parts and layer in just one network, some is about the design of the hub and hub passage in a transport network, but the quantitative studies about the cooperation and interaction between different networks are rare.

Research by Martins de Sá, Contreras and Cordeau (2015) showed an advantage of hub-and-spoke network, hub facilities can be connected with highly efficient pathways, achieving economies of scale on the transportation cost(or travel time)between hubs. Research by O'Kelly (2008) showed that hub location problems consider the design of hub networks by selecting a set of nodes to locate hubs, it activate a set of links and route goods over the network, while optimizing the cost-based (or service-based) objective function. Essentially, the transfer appears to occur within a facility, primarily through the traffic flow of the hub, which is the subject of the analysis.

Facing the competition, logistics enterprises cooperate to optimize the operation network through paid sharing of logistics resources. More research is needed about the cooperation mode of logistics enterprises, management rules and the interactive connection of logistics network. Research by Wang, Ma, Liu, Gong, Liu, Xu et al. (2017) through the existing logistics service providers or participants in the logistics system, and through the negotiation process to organize and cooperate with the two-level logistics joint distribution network, the phenomenon of cross transport can be effectively reduced and the efficiency of urban cargo transport system can be improved. Research by Wang, Zhang, Assogba, Liu, Xu \& Wan (2018) showed the adoption of collaboration strategies among logistics facilities and the formation of one or multiple coalitions constitute a sustainable approach to vehicle routing network optimization. Research by Long (2016) showed the inter-organizational collaboration of supply chain network is an important modern business model. The model includes collaboration and decentralized decision making among different organizations to improve the overall performance of the supply chain network. they studied the principles of inter-organizational collaboration, and proposed a new perspective of collaborative decision-making based on materials, information and time flow.

Our study is about the cooperation between two independent hub-and-spoke logistics networks, they work in coordination to solve the problem that the flow of goods to the hubs was so great that the goods can not be handled in time.

\section{Literature Review}

\subsection{Hub-and-Spoke Network}

The research on the hub-and-spoke network can be carried out from various perspectives, such as the lowest total cost, the shortest path, the shortest time and so on. The methods to solve these problems include accurate calculation model, optimization algorithm and so on. Facilities and transportation links are incorporate in a logistics network which designed by hub-spoke-network. Research by Alibeyg, Contreras and Fernández (2016) showed location decisions focus on selecting a set of nodes to place the central facility, network design decisions deal with the selection of links to the source and destination, possibly through the hub, and the routing of goods through the network. Research by Kian and Kargar (2016) proposed the problem of hub location with power law congestion cost. It is usually convenient to design the network with hub-and-spoke structure, to route traffic in these networks, using hub points and replace direct connections with fewer indirect ones. Research by Ishfaq and Sox (2012) showed in a multimodal transport hub network, cost-effectiveness can be achieved through economies of scale resulting from the use of multimodal transport and the consolidation of cargo flows in the hub. Research by Kartal, Hasgul and Ernst, (2017) showed a single allocation p-hub median location and routing problem with simultaneous pick-up and delivery based on observations of real-life hub networks, the goal is to minimize the cost of the flow between hubs and the flow in the routing network. Research by Karimi and Setak (2018) showed in the 
problem of traffic scheduling and hub location routing, the hubs in the network are not completely interconnected, and the goal is to maximize the total traffic and minimize the total fixed cost and routing cost within the predetermined minimum arrival time. Research by Alumur, Nickel, Rohrbeck and Saldanha-da-Gama (2018)showed modeling framework for hub location issues considering hub crowded service time constraints. The service time model takes into account the running time, processing time and the delay caused by hub congestion. Research by Ilić, Urošević, Brimberg and Mladenović (2010) showed a NP hard problem with locating hub facilities in order to minimize the traffic between all origin-destination pairs. And it presented a new general variable neighbourhood search approach for the uncapacitated single allocation p-hub median problem in networks.

\subsection{Network Competition and Cooperation or Coordination}

Research by Cruijssen, Cools and Dullaert (2007) showed among the general logistics service providers, they firmly believe that the potential benefits of horizontal cooperation are to increase their profitability or improve their service quality, found a reliable partner to lead the collaboration and establishing a fair profit distribution mechanism were the obstacles most identified by respondents. Research by Mesa-Arango and Ukkusuri (2015) showed demand clustering in freight logistics network is an important strategic decision for airlines. It is used to integrate new businesses into their networks, detect the underlying economy, optimize their operations, and develop revenue management strategies. Research by Liu, Wang, Shen, Yan and Wei (2018) showed Logistics service supply chain order allocation, including a logistics service integrator and two competing functional logistics service providers, they have participated in the distribution. Research by Hafezalkotob (2017) showed two energy-saving competition and cooperation models of green supply chain are established. It pointed out that stakeholders in the shipping industry, port authorities, shipping companies and port operators often compete and cooperate within an ecosystem. Research by N. Moradinasab (2018) showed the competition and cooperation between supply chains in the multi-objective oil green supply chain with game theory. Research by Changmin Jiang (2017) showed different air-iron cooperation scenarios, establish two possible air-rail partnerships between rail operators and domestic airlines. Research by Cui and Hertz (2011) Described and analyzed the differences of the three basic types of logistics enterprises in core competitiveness and network development, and the influence of these differences. They complement and interact with each other in the logistics supply chain. Research by Defryn and Sörensen (2018) proposed horizontal logistics cooperation, in which multiple companies work together to solve logistics optimization problems. In order to get the benefits of a single partner in the logistics optimization model, a set of goals is set for each partner. The question is whether the optimization process of the collaborative optimization problem should consider only these individual partner goals, or should a set of joint goals be identified first.

Cooperation among logistics networks requires coordination among all aspects of logistics networks. Research by Chu, Feng and Lai (2018) showed in logistics service innovation, external relationships are important sources of resources and knowledge. Drawing on social capital theory and the strategy-structure-performance paradigm, it proposes that "guanxi" (Chinese system of social networks and relationships that facilitates business and other dealings) accelerates the innovation of third-party logistics service providers in China. Research by Long (2017) integrated computational experiment and data analysis, and proposed a methodology for data-driven computational experiments for inter-organizational collaborations in supply chain networks. Research by Yuan and Yu (2018) showed a mathematical model of the location and linkage design of integrated transit hubs with balance constraints, proposed a multi-channel network, club-based infrastructure framework to capture real geographic and administrative functions and improve overall transit-oriented system performance. Research by Meizhu Li (2018) showed relationships existing in networks cannot be used in multi-relationship complex systems. Therefore, a new network model is used to describe the different relationships between the actual systems, such as multi-layer network and network of networks, and a new method to identify different nodes in the network is proposed. Research by Louis M. Shekhtman (2018) showed many complex networks involve significant interdependencies between different systems. They proposed a spatial network of interdependence and how the cascade process affects spatial embedding. Research by Mesa-Arango and Ukkusuri (2015) showed demand aggregation in freight logistics network is an important strategic decision for carriers. It is used to integrate new businesses into its network, identify potential businesses, and optimize its operations. Research by Niknejad and Petrovic (2014) studied the inventory control and production plan optimization of integrated reverse logistics network, and the 
forward path, repair and remanufacturing and two recovery paths and processing methods of integrated reverse logistics network are studied. Research by Wang, Peng et al. (2018) showed through the establishment of cooperative alliance, the optimization of secondary logistics distribution and loading network can be effectively realized. With the coordination of existing network or logistics facilities of logistics service providers, the high operating cost of cross-long-distance transportation can be reduced by inclusive restructuring of the entire network.

\subsection{Genetic Algorithm}

There are a lot of researches on genetic algorithm to solve hub-and-spoke networks, and there are also designs using genetic algorithm in logistics network optimization and supply chain collaboration or coordination, but, not much literature has been found for the design of genetic algorithms for the interaction between two independent hub-and-spoke networks.

Research by Huang and Wang (2009) showed in order to reduce the risk of uncertainty in the process of network optimization, the optimal robust solution of hub-spoke network is obtained under the condition of multiple possibilities of demand and cost, and an optimization method based on multi-objective optimization genetic algorithm is proposed. Research by Gen, Lin, Yun and Inoue (2018) showed the latest progress of genetic algorithm based on hybrid priority in solving multilevel logistics or supply chain management network problems. Introduced: (1) sugar cane supply chain management network model, (2) multi-objective supply chain network model, (3) flexible multistage logistics network model, (4) multi-objective reverse logistics network model. Research by Zhang, Deng, Chan and Zhang (2013) showed a central coordination system framework with multi-criteria genetic optimization. In the previous multi-criteria optimization genetic algorithm (MCOGA), the analytic hierarchy process was used to evaluate the fitness value. And an improved MCOGA algorithm based on order preference technique similar to ideal solution is proposed. Research by Young-Bin Woo (2019) a hydrogen supply chain network problem with two modes of transport and supply cycles. Mixed integer linear programming is introduced in the process of genetic algorithm, and a mathematical model based on genetic algorithm is proposed. Research by Delavar, Hajiaghaei-Keshteli and Molla-Alizadeh-Zavardehi (2010) showed a major issue in supply chain management is the coordination of production and distribution decisions. To achieve effective logistics scheduling, the key is to integrate these two functions and coordinate planning. Taguchi experimental design method is applied to set and estimate the proper values of genetic algorithm parameters to improve the performance.

\section{Problem and Methods}

The research explores ways for cooperation between two hub-and-spoke logistics networks of company A and B. Our object is to find which of the hubs in primary network that requires shunting and which of the hubs in cooperation network can cooperate to minimize the cost between the cooperation of the two networks. Each company has its independent network, different outlets and processing capacity of hub. The outlets which form the network come from three provinces in northern China and another three provinces in southern China of company A and B. We assume that network A(B) of overloaded traffic in hub facing a risk that the flow of incoming goods in the hubs exceeds handling ability and needs shunting, the cooperation network $\mathrm{B}(\mathrm{A})$ has sufficient processing power to handle these goods shunted to it, and they can co-utilize the resources of the two logistics networks. At first, the hub-and-spoke logistics networks of the two companies were constructed according to the actual data. The total cargo flow arrive to these hubs in network A and B is calculated, then we set that the top $N$ hubs in total cargo flow ranking in one network may face the risk of not being able to handle the flow of goods in time, and the network need to shunt some traffic from these hubs to hubs in another company's network. So, the flow shunts from one logistics network to another leads to the interaction and cooperation of the two hub-and-spoke networks. The hubs from cooperation network collaborated to shunt the cargo flow. When these flows are shunted to network, they will be distributed to the corresponding destination just like other flows, and the hub-and-spoke logistics networks of $\mathrm{A}$ and $\mathrm{B}$ will remain in operation.

Our innovation lies in the application of quantitative method to study the cooperation and resource integration of two logistics networks, the problem that logistics companies can't deal with goods in time in the peak season is solved by means of shunt. 
In section 4 we build a descriptive model of the problem and then design a multilevel genetic algorithm to solve the problem. This model gives us a basic description of the problem, and reflects our basic understanding of the problem, but it is not a precise expression of the problem, and we do not have a precise solution of the model. To solve this problem, we design the corresponding genetic algorithm in in section 5. In the model, both logistics networks are designed with a single allocation hub-and-spoke network then we extend it according to the characteristics of the new problem. When the model is built, it is assumed that A company will shunt the goods to B company, if the goods are shunted from B company to A company, the model principle is the same.

\section{Mathematical Formulation}

Although the two companies have the same hub-and-spoke network model, but when design the network, it must use their actual parameters respectively. There are different parameters of network A and B, such as the node's longitude and latitude coordinates, here are some instructions of variables and parameters in the Mathematical Formulation.

\begin{tabular}{|c|c|}
\hline \multicolumn{2}{|l|}{ Index: } \\
\hline$n$ & The nodes in network A \\
\hline$m$ & The nodes in network B \\
\hline$N$ & $\begin{array}{l}\text { The number of top } N \text { hubs in total cargo flow ranking, and the total cargo flow arrive to the hub in } \\
\text { network A }\end{array}$ \\
\hline$q \in\{1,2,3, \ldots N\}$ & The qth hub in the $N$ hubs \\
\hline \multicolumn{2}{|l|}{ Parameters: } \\
\hline$C^{c}$ & Collection cost per unit distance per flow, in network A and network B \\
\hline$C^{d}$ & Distribution cost per unit distance per flow, in network A and network B \\
\hline$C^{t}$ & Transport cost per unit distance between two hub points, in network A and network B \\
\hline$f_{k}$ & The cost of building a hub on a node, in network $\mathrm{A}$ and network $\mathrm{B}$ \\
\hline$D_{i j}^{A}$ & Distance matrix in network $A, i, j \in\{1,2, \ldots n\}$ \\
\hline$D_{\mathrm{ij}}^{B}$ & Distance matrix in network $\mathrm{B}, i, j \in\{1,2, \ldots m\}$ \\
\hline$D_{k_{q} v}^{A B}$ & $\begin{array}{l}\text { The distance between } q \text { th shunting hub } k \text { in network } A \text { and cooperation hub } v \text { in network } B \text {, } \\
k \in\{1,2, \ldots n\}, v \in\{1,2, \ldots m\}, q \in\{1,2, \ldots N\}\end{array}$ \\
\hline$W_{i j}^{A}$ & Traffic from point $i$ to $j$ point in network $A, i, j \in\{1,2, \ldots n\}$ \\
\hline$W_{i j}^{B}$ & Traffic from point $i$ to point $j$ in network $\mathrm{B}, i, j \in\{1,2, \ldots m\}$ \\
\hline$O_{i}^{B}$ & $\begin{array}{l}\text { The total amount of flow departing from point } i \text {, in network A; } \\
O_{i}^{A}=\sum_{j} w_{i j}^{A}, i, j \in\{1,2, \ldots n\}\end{array}$ \\
\hline$O_{i}^{B}$ & $\begin{array}{l}\text { The total amount of flow departing from point } i \text {, in network B; } \\
O^{B}{ }_{i}=\sum_{i} w^{B}{ }_{i j}, i, j \in\{1,2, \ldots m\}\end{array}$ \\
\hline$Q_{i}^{A}$ & $\begin{array}{l}\text { The total amount of cargo in network } A \text { that arrived to } i \text {, } \\
Q_{i}^{A}=\sum_{j} w_{j i}, i, j \in\{1,2, \ldots n\}\end{array}$ \\
\hline$Q_{i}^{B}$ & $\begin{array}{l}\text { The total amount of cargo in network B that arrived to } i \text {, } \\
Q_{i}^{B}=\sum_{j} w_{j i}, i, j \in\{1,2, \ldots n\}\end{array}$ \\
\hline$x_{A}, y_{A}$ & Node coordinate in network $\mathrm{A}, x_{A}$ is abscissa, $y_{A}$ is vertical coordinates \\
\hline
\end{tabular}




\begin{tabular}{|c|c|}
\hline$x_{B}, y_{B}$ & Node coordinate in network $\mathrm{B}, x_{B}$ is abscissa, $y_{B}$ is vertical coordinates \\
\hline$\alpha$ & $\begin{array}{l}\text { The transport parameters between two networks account for the proportion of the transport } \\
\text { parameters between hubs in one hub-and-spoke network }\end{array}$ \\
\hline$\beta$ & A percentage of the shunt of the cargo flow which arrive at the hub point \\
\hline G & $i$ rows and $j$ columns matrix that corresponds to a uniform \\
\hline \multicolumn{2}{|l|}{ Variables: } \\
\hline$x_{i k} \in\{0,1\}$ & $\begin{array}{l}\text { When } i=k, x_{k k}=1 \text { it means } k \text { is hub point. When } i \neq k \text { and } x_{i k}=1 \text {, it means radial node } i \text { is } \\
\text { assigned to the hub point } k \text {, otherwise, } x_{i k}=0, i, k \in\{1,2, \ldots n\}\end{array}$ \\
\hline$y_{i k l} \geq 0$ & Flow of goods from point $i$ through hub $k$ and $l, i, k, l \in\{1,2, \ldots n\}$ \\
\hline$u_{i k_{q}}^{A} \geq 0$ & $\begin{array}{l}\text { In the network } A \text { the flow from node } i \text { arrive to } q \text { th hub } k \text {, and hub } k \text { is among the top } N \text { hubs in } \\
\text { total cargo flow ranking, and the total cargo flow arrive to the hub in network } A, i, k \in\{1,2, \ldots n\}, q \\
\in\{1,2, \ldots N\}\end{array}$ \\
\hline$u_{i k}^{B} \geq 0$ & The flow from node $i$ and reach hub $k$ in the network $\mathrm{B}, i, k \in\{1,2, \ldots m\}$ \\
\hline$z_{k_{q} v}^{A B} \in\{0,1\}$ & $\begin{array}{l}\text { In network } \mathrm{A} \text { the hub } k \text { choose hub } v \text { in network B for stunting cooperation. } \\
k \in\{1,2, \ldots, \mathrm{n}\}, v \in\{1,2, \ldots \mathrm{m}\}, q \in\{1,2, \ldots N\}\end{array}$ \\
\hline$h_{i k_{q}}^{A}$ & $\begin{array}{l}\text { The cargo flow arrive to } q \text { th hub } k \text { after stunting in network A, } \\
k \in\{1,2, \ldots, \mathrm{n}\}, i \in\{1,2, \ldots n\}, q \in\{1,2, \ldots N\}\end{array}$ \\
\hline$h_{i v}^{B}$ & $\begin{array}{l}\text { The total cargo flow arrive to hub } k \text { in network B after it receive the stunting cargo flow from A, } \\
i \in\{1,2, \ldots m\}, v \in\{1,2, \ldots \mathrm{m}\}\end{array}$ \\
\hline
\end{tabular}

Descriptive mathematical model:

$$
\begin{gathered}
\min F=f^{A}+f^{B}+T_{A B} \\
f^{A}=f^{f}+f^{c}+f^{d}+f^{t} \\
f^{f}=\sum_{k} f_{k} x_{k k} \\
f^{c}=\sum_{i k} C^{c} D_{i k} O_{i} x_{i k} \\
f^{d}=\sum_{i k} C^{d} D_{k i} Q_{i} x_{i k} \\
f^{t}=\sum_{i k l} C^{t} D_{k l} y_{i k l} \\
\sum_{k} x_{i k}=1, \forall i \\
x_{i k} \leq x_{k k}, \forall i, k \\
\sum_{l} y_{i k l}-\sum_{l} y_{i l k}=O_{i} x_{i k}-\sum_{l} W_{i j} x_{j k}, \forall i, k \\
\sum_{l, k \neq l} y_{i k l} \leq O_{i} x_{i k}, \forall i, k \\
\sum_{i}, \forall i, k
\end{gathered}
$$




$$
\begin{aligned}
& y_{i k l} \geq 0, \forall i, k, l \\
& T_{A B}=\alpha C^{t} \mathrm{D}_{A B} \beta u_{i k_{q}}^{A} \cdot z_{k_{q} v}^{A B}, \forall i, k, q, v \\
& u_{i k_{q}}^{A}=\sum_{l} \sum_{i}\left(w_{i k_{q}}^{A}+y_{l k_{q}}^{A B}\right) \cdot x_{i k_{q}}, \forall i, k, q \\
& u_{i k}^{B}=\sum_{l} \sum_{i}\left(w_{i k}^{B}+y_{l k}^{B}\right) \cdot x_{i k}, \forall i, k \\
& h_{i k_{q}}^{A}=u_{i k_{q}}^{A}\left[1-\alpha u_{i k_{q}}^{A} \cdot z_{k_{q} v}^{A B}\right], \forall i, k, q \\
& h_{i k_{q}}^{B}=u_{i k}^{B}\left[1+\alpha u_{i k_{q}}^{A} \cdot z_{k_{q} v}^{A B}\right], \forall i, k, q \\
& \sum_{l} y_{\mathrm{ik}_{q} l}^{A}-\sum_{l} y_{i l k_{q}}^{A}=h_{i k_{q}}^{A} \cdot x_{i k_{q}}^{A}-\sum_{l} W_{i j}^{A} x_{j k_{q}}^{A}, \forall i, k, q \\
& \sum_{l, k \neq l} y_{i k_{q} l}^{A} \leq h_{i k_{q}}^{A} \cdot x_{i k_{q}}^{A}, \forall i, k, q \\
& \sum_{l} y_{\mathrm{ik}_{q} l}^{B}-\sum_{l} y_{i l k_{q}}^{B}=h_{i k_{q}}^{B} \cdot x_{i k_{q}}^{B}-\sum_{l} W_{i j} x_{j k_{q}}^{B}, \forall i, j, k, l, q \\
& \sum_{l, k \neq l} y_{i k_{q} l}^{B} \leq h_{i k_{q}}^{B} \cdot x_{i k_{q}}^{B}, \forall i, k, l, q \\
& y_{l k_{q}}^{A B}=\alpha \cdot u_{i k_{q}}^{A}, \forall k, l, q \\
& z_{k_{q} v}^{A B} \in\{0,1\}, \forall k, v, q \\
& u_{i k_{q}}^{A} \geq 0, u_{i k}^{B} \geq 0, h_{i k_{q}}^{A} \geq 0, h_{i k_{q}}^{B} \geq 0, y_{l k_{q}}^{A B} \geq 0 \forall i, \mathrm{k}, v, q
\end{aligned}
$$

Equation (1) the total cost in the question composed of the construction cost of hub-and-spoke network $\mathrm{A}$ and that of network B and the shunt cost between two hub-and-spoke networks as shown in equation. Equation (2) the $\operatorname{cost} f^{A}$ is the construction cost of network $\mathrm{A}$, and so is $f^{B}$ in network $\mathrm{B}$. It includes four parts, which are: construction cost of hub point (equation (3)). The collection cost in the process of transporting the goods from the starting node to the hub point (Equation (4)). Distribution cost from the hub point to the destination node (Equation (5)). Transportation cost of main line (Equation (6)); Equation (7), (8) is the constraint of node allocation relation, indicating that each node can only be used as a hub node or a hub's radial nodes, and cannot exist independently. It means that only when $x_{k,}$ is 1 , i.e. the node $k$ is selected as the hub node, can other nodes be allocated to the node; otherwise, node $k$ cannot have the radial node. Equation (9) is the balance constraint of the node's cargo flow. When a point is a hub point, there is a volume flow between the hubs, otherwise 0 (Equation (10)). (11), (12) properties that constrain variables.

Equation (13) means the shunt cost which is the transfer cost between the hubs in network $\mathrm{A}$ and the hubs in network B. Equation (14) the total cargo traffic that arrive at hub $k$ which belong to the $N$ hub points in network A, $N$ means top $N$ in the cargo traffic ranking that arrive at all the hub points. Equation (15) in network B the total traffic that arrive at $q$ th hub k. This goods flow consists of two parts, flow from other hub points and the flow from one of its corresponding radial nodes. Equation (16) when the hub $k$ in network A cooperates with the hub $v$ 
from network $\mathrm{B}$, the traffic that originally arrived at hub $k$ in network $\mathrm{A}$ is shunted to hub $v$. Equation (17) When the hub $k$ in network A cooperates with the hub $v$ from network B, flow to hub $v$ in network B increased by the traffic from hub $k$ in network A. Equation (18) and (19) means that in network A after the traffic in hub $k$ is shunted, this hub-and-spoke network still follows the constraint of traffic balance, resume normal operation. Equation (20) and (21) means that in network B after the traffic in hub $v$ increased by the flow from hub $k$ in network $\mathrm{A}$, this hub-and-spoke network still follows the constraint of traffic balance, and the traffic that come from network A will be distributed to the destination by network B. Equation (22). The cargo traffic transferred between network A and network B is equal to the traffic diverted from the hub of network A. (23), (24) properties that constrain variables.

\section{Multilayer Genetic Algorithm \\ 5.1. Algorithm Design}

Research by Shi, Wang and Yu (2011) showed that the multi-level coding genetic algorithm divides the individual coding into several levels, each of which represents different meanings, and the multi-level coding fully expresses the solution of the problem, thus accurately expressing the solution of the complex problem with one chromosome. Because there are two independent logistics networks in the calculation, and we study the cooperation of two independent logistics networks, in algorithm design, we use multi-layer coding genetic algorithm.

In the multi-level coding genetic algorithm, one chromosome is divided into two parts in coding by the gene respectively representing primary network $\mathrm{A}(\mathrm{B})$ and cooperation network $\mathrm{B}(\mathrm{A})$, in which, $\mathrm{B}$ is capable of handling the cargo shunted from A or viceversa. When crossing, mutating and decoding, the gene of two networks should also be clearly distinguished. Perform algebra is 200; Chromosome population size is 60 .

Coding: The encoding is permutation encoding, we arrange the genes on the chromosomes, and each crossover mutation randomly shuffles the arrangement of the genome to produce different chromosomes. The locus of 1 means that this point is hub, the locus of 0 means that this point is non-hub, see Figure 1. Each chromosome is divided into two parts, which are the coding of network A and network B respectively. The number of genes in each part of coding is the total number of nodes in this network. The number of genes in chromosomes: the scale of the genes in the southern region is 2299 , in which the number of genes in network A is 1781 and in network B is 518. In the northern region, the total the scale of genes is 1694, in which the number of genes of network A is 912 and the number of genes of network B is 782 .

Crossover: see Figure 1. We choose multi-point crossing, there are nearly a thousand gene positions on a chromosome, multi-point crossing can make multiple gene positions cross each other quickly, two chromosomes are randomly selected to cross, and the positions of genes on the chromosomes are multiplied, changing the arrangement of $0 \backslash 1$ genes on the chromosomes, so as to form a new chromosome. The crossover rate is 0.7 , because the greater the crossover probability, the faster the new individuals will be produced, and the more chromosomes will be involved in the crossover in a short period of time, however, when the crossover probability is too large, the probability of the genetic model being destroyed may also be higher. The two different parts of the chromosome code do not cross each other and ensure that the number of hub points expressed by the segments of the chromosome representing network A and network B remains constant.

Mutate: the mutate method is multi-point variation, the mutation is carried out by changing the position of the original gene in the chromosome, to replace the value of a gene at a locus in the coding sequence of an individual chromosome with another allele at that locus. For the mutation probability, if the value is too small, it is not easy to generate new individual structure; if the value is too large, then the genetic algorithm becomes a pure random search algorithm, mutation probability is 0.4 . For the mutation ensure that the number of hub points expressed by the segments of the chromosome representing network A and network B remains constant.

Decoding: in decoding, the hub points selected from the chromosome are allocated to the appropriate spoke node, we respectively construct two logistics networks in the three northern provinces and the southern provinces by genetic algorithm toolbox, then we calculate the total cargo flow arrive to each hub point in network A and 
network. At last we select the top $\mathrm{N}$ hubs in total cargo flow ranking, that need to be shunted in network $\mathrm{A}$, and then choose the hub points that are in cooperation with shunted hubs in network A from network B.

Fitness: the fitness function is Equation (1), calculate the minimum cost.

Genetic operation: the selection operation adopts the roulette method

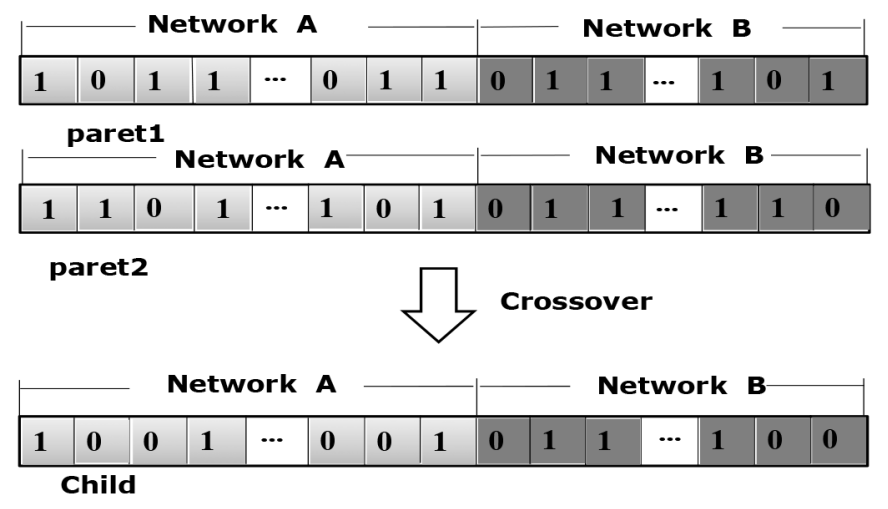

Figure 1. Genetic algorithm multipoint crossover

\subsection{Sample and Analysis}

The data set in the algorithm comes from two Chinese third-party logistics companies A and B, and we select their outlets in three provinces in north China (Beijing, Tianjin, Hebei) and three provinces in south China (Jiangsu, Zhejiang, Shanghai) respectively, the three northern provinces are regionally adjacent to each other, as are the three southern provinces. In the northern region, company A shunt flow to company B, and in the southern region, company B shunt flow to company A.

In both the southern region and the northern region, we select $N$ hubs from primary network that need to be shunted, and then calculated the hub points in cooperation network. Transport parameters between two networks is $\alpha \cdot C$. Parameter sensitivity analysis was performed, keep the other parameters constant, let the parameter $r$ and $\alpha$ change, to analyze the influence of traffic flow in each hub and spoke network and the transport cost between the two networks on the selection of hub that need shunted and cooperation hub. $u_{i k_{q}}^{A}$ means hubs need to be shunted in network A, $z_{k_{q} v}^{A B}$ means hubs that choose from network B to cooperation with network A, and there is a one-toone correspondence between cooperation hubs and hubs requiring shunting. $u_{i k_{q}}^{B}$ means hubs need to be shunted in network $\mathrm{B}, z_{k_{q} v}^{B A}$ means hubs that are chosen from network A to cooperation with network B. For the calculation in the southern region or the northern region when the same parameter setting occurs, the calculation results will be different at different times, for example, in table 2, when $\alpha$ is fixed to 10 and the parameter $r$ is changed to 1 , the calculation result is different from when $r$ is fixed to 1 and $\alpha$ is changed to 10 , because the heuristic algorithm has certain randomness.

The flow of goods in the network is calculated from economic gravity models, data comes from the companies published on the internet and "China economic statistical yearbook 2017". Research by Ping (2014) showed us the parameters of the economic gravity model in this manuscript, so the calculation of the flow between nodes in the logistics network is (25).

$$
\ln M_{i j}=-2.77+0.64 \ln \left(Y_{i} Y_{j}\right)-0.55 \ln D_{i j}-0.12 e_{i j}+\varepsilon_{i j}
$$

In the Economic Gravity Model (25), the value of $\delta_{1}$ is 0.64 indicates that when the product of land $i$ and land $j$ GDP increases by $1 \%$, the trade flow between them will rise by $0.64 \%$. In other words, the more developed the economy of the two places, the more beneficial the increase of the trade volume of the two places will be. The 
value of $\delta_{2}$ is -0.55 , which means that when the distance between land $i$ and land $j$ increases by $1 \%$, the trade flow from land to land decreases by $0.55 \%$. In other words, the greater the distance between the two sides, the more unfavorable the trade exchange between the two places. $\delta_{3}$ indicating that when the country is not part of the China-ASEAN free trade area, China's imports from the country will decrease by $\delta_{3} \%$, $e_{i j}$ is a dummy variable, in this case, we set $\delta_{3}$ is 0 , and $e_{i j}$ is 0 . Because the flow calculated by the economic gravity model is symmetric, and these flow are not symmetric in the actual logistics operation, the flow matrix is multiplied by a matrix of $G$, let $G$ be a $i$ rows and $j$ columns matrix that corresponds to a uniform distribution between 0 and 1 , traffic between nodes $W_{i j}=G \cdot M_{i j}$, then the flow matrix is obtained.

All the calculation examples are carried out in MATLAB R2016a, is the number of hubs in network A, is the number of hubs in network B; is the number of hubs that need to shunt traffic. The fixed parameter values in the sample are in Table 1.

\begin{tabular}{|c|c|c|c|c|}
\hline$C^{c}$ & $C^{d}$ & $C$ & $\begin{array}{c}p^{A} \text { in northern } \\
\text { region }\end{array}$ & $\begin{array}{c}p^{B} \text { in northern } \\
\text { region }\end{array}$ \\
\hline 1.5 & 2 & 0.55 & 80 & 50 \\
\hline $\begin{array}{c}p^{A} \text { in southern } \\
\text { region }\end{array}$ & $\begin{array}{c}p^{B} \text { in southern } \\
\text { region }\end{array}$ & $\begin{array}{c}\text { Nin northern } \\
\text { region }\end{array}$ & $\begin{array}{c}\text { Nin southern } \\
\text { region }\end{array}$ & $f_{k}$ \\
\hline 120 & 30 & 20 & 15 & 0 \\
\hline
\end{tabular}

Table 1. The fixed parameter values

\subsubsection{Analysis in Northern Region}

At first we analyze the result in northern region, the number of nodes in network A is 912 and the number of nodes in network B is 782. Network A need to shunt the flow and network B is a cooperative network. The calculation results of node data in the northern region are shown in Table 2, Figures 2, 3, 4 and 5.

In Table 2, keep the other parameters constant, let the parameter $r$ change from small to large to adjust the size of the flow matrix. When the value of $r$ increases, the traffic of the corresponding network also increases and generally speaking, the traffic in the network increases the traffic flowing through the hub point also increases. The hubs with the largest traffic in network A need to be shunted to the hubs in cooperative network $\mathrm{B}$, the total cost $F$ is lowest when the flow parameter $r$ are lowest, the increase of network traffic leads to the change of hub points that need to be shunted, and so the cooperative hubs $z_{k_{q} v^{*}}^{A B}$ This result shows that the size of network traffic affects the choice of hubs requiring shunting and cooperation.

In Table 2, keep the other parameters constant, let another parameter $\alpha$ change from small to large, $\alpha \cdot c^{t}$ also change from small to large, so the transport cost between network A and network B is increasing. The shunted hub points $u_{i k_{q}}^{A}$ change according to the change of $\alpha$, and so the cooperative hubs. This result shows that the transport cost between network A and network B affects the choice of hubs requiring shunting and cooperation hubs.

In Figure 2 the convergence curve of genetic algorithm converges slowly, the optimal value curve and the average value curve tend to coincide at the end of the algorithm iteration. In Figure 3 and Figure 4 we can see that, when the value of $\alpha$ changes from 0.5 to 15 the structure of the hub-and-spoke network of $\mathrm{A}$ and $\mathrm{B}$ changed. Because of the multi-layer coding method in genetic algorithm, one chromosome carries the information of network $\mathrm{A}$ and network B at the same time, the hub and spoke network graph of network A and network B overlapped in the same region is obtained at the end of algorithm, see Figure 5. 


\begin{tabular}{|c|c|c|c|}
\hline $\begin{array}{l}\text { Variable parameter } \\
\text { is } r \text {, when } \alpha=10\end{array}$ & $u_{i k_{q}}^{A}$ & $z_{k_{q} v}^{A B}$ & $F$ \\
\hline$r=0.001$ & $\begin{array}{l}\text { 140,123,98,870,242,199,13,210,176,826, } \\
277,91,844,216,170,841,57,741,233,359\end{array}$ & $\begin{array}{l}\text { 139,141,141,742,52,139,439,52,139,768, } \\
34,185,768,77,165,742,99,752,95,530\end{array}$ & $3.2234 \mathrm{e}+10$ \\
\hline$r=0.01$ & $\begin{array}{l}829,691,867,87,164,141,258,198,243,90 \\
795,743,159,178,863,68,247,769,42,266\end{array}$ & $\begin{array}{l}\text { 768,460,737,202,129,139,213,170,170,202, } \\
768,732,170,139,722,19,235,731,6,235\end{array}$ & $3.2616 \mathrm{e}+11$ \\
\hline$r=1$ & $\begin{array}{l}837,207,171,87,872,110,69,117,251,124 \\
838,224,356,791,163,750,42,278,262,653\end{array}$ & $\begin{array}{l}723,75,117,167,703,141,190,211,60,211 \\
709,49,518,724,211,760,16,138,138,429\end{array}$ & $3.3342 \mathrm{e}+13$ \\
\hline$r=5$ & $\begin{array}{l}155,225,141,839,99,851,205,78,876,700 \\
811,358,666,65,277,32,778,748,795,55\end{array}$ & $\begin{array}{l}214,78,203,768,203,768,62,212,704,433 \\
768,564,433,176,35,18,689,756,768,17\end{array}$ & $1.6546 \mathrm{e}+14$ \\
\hline$r=10$ & $\begin{array}{l}691,813,200,167,83,138,90,258,848,243 \\
861,358,121,149,35,748,208111,50,834\end{array}$ & $\begin{array}{l}427,761,170,139,176,139,172,115,761,58 \\
761,545,176,205,148,758,68,176,148,761\end{array}$ & $3.3030 \mathrm{e}+14$ \\
\hline \multicolumn{4}{|l|}{$\begin{array}{l}\text { Variable parameter } \\
\text { is } \alpha \text {, when } r=1\end{array}$} \\
\hline$\alpha=0.5$ & $\begin{array}{l}164,868,671,225,123,822,46,187,360,227 \\
795,77,91,102,65,271,212,749,283,885\end{array}$ & $\begin{array}{l}210,747,429,73,191,747,747,73,547,73 \\
730,157,202,157,210,138,138,692,221,773\end{array}$ & $3.3085 e+13$ \\
\hline$\alpha=2$ & $\begin{array}{l}687,228,122,872,116,76,271,204,795,140 \\
208,194,846,843,746,42,152,50,821,126\end{array}$ & $\begin{array}{l}\text { 433,73,147,737,202,158,123,73,761,134, } \\
222,134,761,746,756,158,134,158,761,147\end{array}$ & $3.2821 \mathrm{e}+13$ \\
\hline$\alpha=5$ & $\begin{array}{l}141,147,238,858,832,221,72,189,682,76 \\
261,829,347,744,216,268,41,824,75,107\end{array}$ & $\begin{array}{l}208,129,54,688,688,62,105,129,445,189 \\
62,688,546,714,54,64,6,699,189,201\end{array}$ & $3.3080 \mathrm{e}+13$ \\
\hline$\alpha=10$ & $\begin{array}{l}\text { 179,862,122,242,207,121,826,80,213,845, } \\
682,252,753,350,90,62,814,54,277,30\end{array}$ & $\begin{array}{l}\text { 130,744,191,43,170,210,743,172,225,744, } \\
459,127,687,521,172,172,730,107,127,13\end{array}$ & $3.2931 \mathrm{e}+13$ \\
\hline$\alpha=15$ & $\begin{array}{l}822,689,176,862,257,855,132,87,193,242 \\
277,752,207,111,109,68,39,97,170,29\end{array}$ & $\begin{array}{l}696,434,119,741,54,741,211,211,74,74,72, \\
715,74,211,206,149,254,211,206,161\end{array}$ & $3.3241 e+13$ \\
\hline
\end{tabular}

Table 2. Experimental results in the northern region

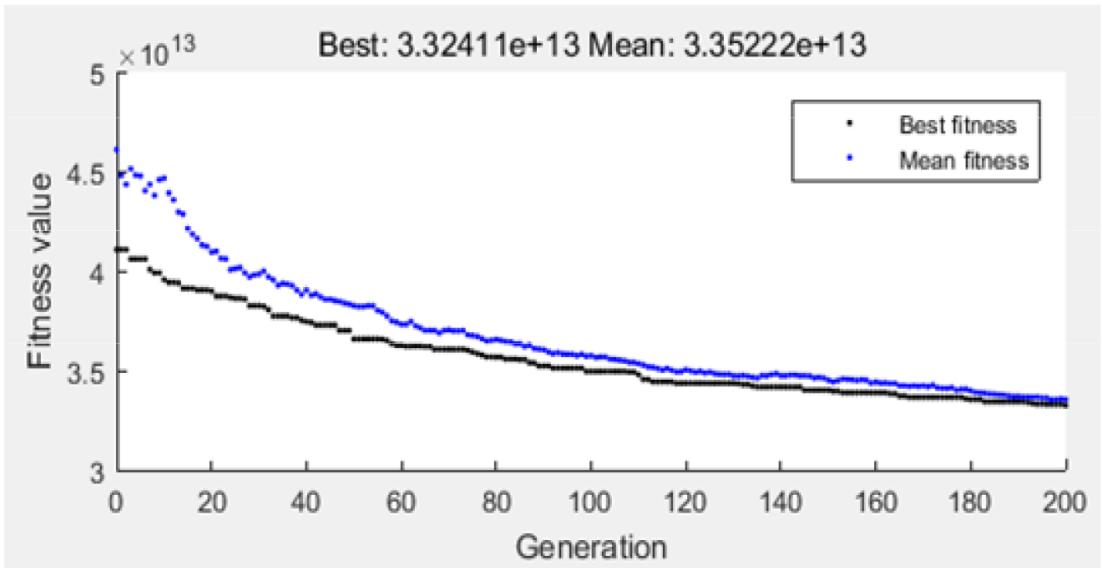

Figure 2. Convergence curve of genetic algorithm 


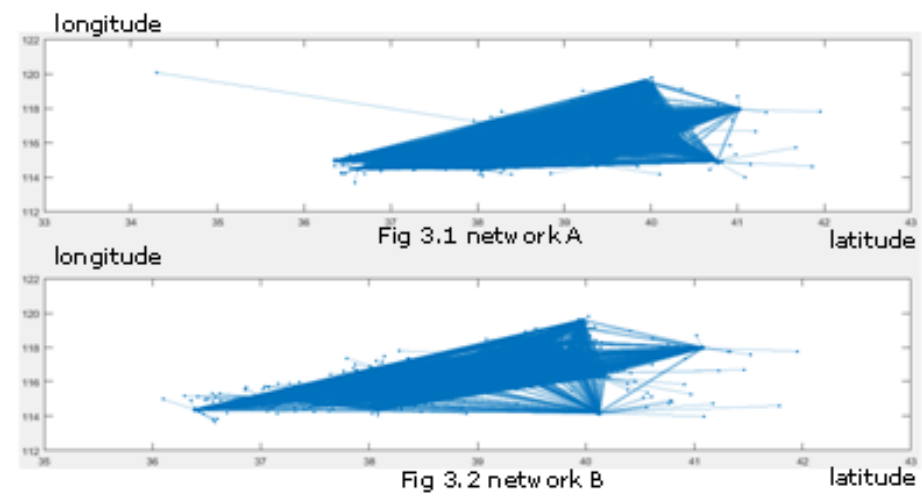

Hub of network $A$ and $B ; O$ spoke of network $A$ and $B$

Link of hub and spoke, link of hub and hub in network $A$ and $B$

Figure 3. The hub and spoke network in the northern region, $r=1, \alpha=0.5$

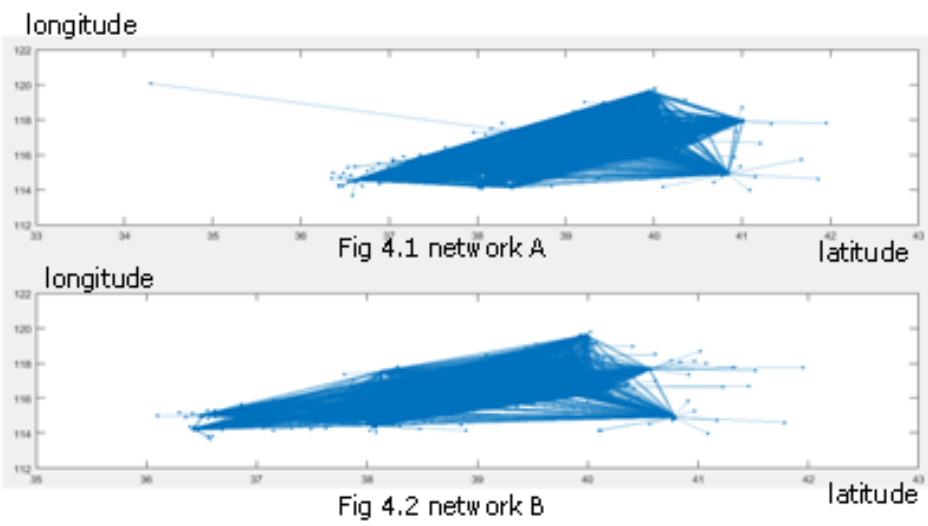

Hub of network $A$ and $B ; O$ spoke of network $A$ and $B$

_ Link of hub and spoke, link of hub and hub in network A and B

Figure 4. The hub and spoke network in the northern region $r=1, \alpha=15$

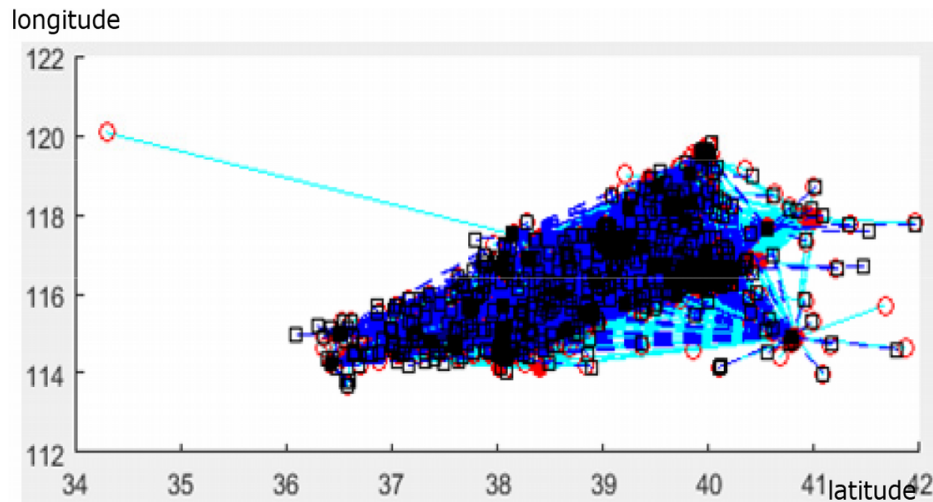

hub of network A Ospoke of network A

The link of hub and spoke, link of hub and hub in network $A$

Hub of network B $\square$ spoke of network B

- - 'The link of hub and spoke, link of hub and hub of network $B$

Figure 5. Two overlapping hub- and-spoke network of A and B in the northern region $r=1, \alpha=15$ 


\subsubsection{Analysis in Northern Region}

Next, we analyze the result in southern region. We set up network A to shunt goods to network B so network B is a cooperative network. The number of nodes in network A is 1781 and the number of nodes in network B is 518, there is a large gap in the number of outlets between A and B. Keep the other parameters constant, let the parameter $\alpha$ and $r$ change from small to large, to analyze the influence of flow in each network and the transport cost between the two networks on the selection of hub that need shunting the flow and cooperation hub. Table 3 , Figure 6, Figure 7 and Figure 8 show the calculation results and it shows that the sensitivity analysis results of parameters in the southern region are consistent with those in the northern region. This result shows that the size of network cargo flow and the transport cost between network A and network B affects the choice of hubs requiring shunting and its cooperation hubs.

In Figure 6, when the value of $\alpha$ is 15, the mean curve of genetic algorithm doesn't converge to the best value curve but it keeps going to the optimal value, the convergence of the curve is not ideal compared with the calculation in the northern region. This is mainly because the calculated data scale of network A in southern region is relatively large, the mean curve and the optimal value curve cannot be fitted within the set number of iterations. From Figure 7, we can see that network A and network B have different hub-and-spoke network graphs, and network A has significantly more nodes than network B. When network B has a peak of cargo flow and some hubs need to shunt traffic, network $A$ can take advantage of its comprehensive network coverage to carry out paid cooperation with network B on logistics resources. At the end, the hub-and-spoke network graph of network A and network B overlapped in the same region is obtained in Figure 8.

\begin{tabular}{|c|c|c|c|}
\hline $\begin{array}{l}\text { Variable parameter } \\
\text { is } r \text {, when } \alpha=10\end{array}$ & $u_{i k_{q}}^{B}$ & $z_{k_{q} v}^{B A}$ & $F$ \\
\hline$r=0.001$ & $\begin{array}{l}97,192,261,168,226,141,155,35,26,67,247, \\
329,69,182,489\end{array}$ & $\begin{array}{l}\text { 144,50,83,200,51,83,166,146,183,80,148, } \\
1033,64,80,277\end{array}$ & $2.0093 \mathrm{e}+11$ \\
\hline$r=0.01$ & $\begin{array}{l}\text { 130,53,167,69,52,103,226,43,239,7,341, } \\
188,145,168,454\end{array}$ & $\begin{array}{l}150,125,167,90,77,176,50,77,50,201,1022, \\
90,125,176,800\end{array}$ & $2.0577 \mathrm{e}+12$ \\
\hline$r=1$ & $\begin{array}{l}145,17,111,90,146,227,157,234,88,342,22 \\
219,190,56,331\end{array}$ & $\begin{array}{l}\text { 106,154,179,154,125,55,179,16,150,1014, } \\
154,55,2,154,1143\end{array}$ & $2.0298 \mathrm{e}+14$ \\
\hline$r=5$ & $\begin{array}{l}\text { 41,264,88,269,114,39,14,47,220,169,108, } \\
237,175,491,248\end{array}$ & $\begin{array}{l}\text { 131,95,144,89,172,183,183,137,22,193, } \\
184,11,22,290,44\end{array}$ & $1.0143 e+15$ \\
\hline$r=10$ & $\begin{array}{l}46,75,34,143,105,97,171,235,233,171,235 \\
233,77,242,11,117,317,32\end{array}$ & $\begin{array}{l}77,155,155,150,176,150,198,31,77,77,176 \\
155,176,1188,203\end{array}$ & $1.9553 e+15$ \\
\hline \multicolumn{4}{|l|}{$\begin{array}{l}\text { Variable parameter } \\
\text { is } \alpha \text {, when } r=1\end{array}$} \\
\hline$\alpha=0.5$ & $\begin{array}{l}130,266,204,17,48,145,231,29,127,105,12 \\
77,123,415,318\end{array}$ & $\begin{array}{l}129,91,43,140,124,129,35,140,129,129, \\
140,35,198,632,1226\end{array}$ & $2.0447 \mathrm{e}+14$ \\
\hline$\alpha=2$ & $\begin{array}{l}26,37,93,129,267,205,275,210,148,184,249, \\
257,337,159,343\end{array}$ & $\begin{array}{l}\text { 183,164,134,188,76,45,76,37,134,22,134, } \\
45,1028,1019,1028\end{array}$ & $2.0101 \mathrm{e}+14$ \\
\hline$\alpha=5$ & $\begin{array}{l}265,196,85,66,110,20,155,215,79,253,56, \\
113,173,159,293\end{array}$ & $\begin{array}{l}\text { 74,42,132,98,144,183,183,59,132,74,98, } \\
145,98,202,1237\end{array}$ & $2.0345 \mathrm{e}+14$ \\
\hline$\alpha=10$ & $\begin{array}{l}87,55,95,107,127,146,257,231,278,253,240, \\
333,171,84,194\end{array}$ & $\begin{array}{l}131,155,110,144,44,144,77,56,131,110,12 \\
1022,202,56,32\end{array}$ & $2.0430 \mathrm{e}+14$ \\
\hline$\alpha=15$ & $\begin{array}{l}214,130,54,157,314,78,103,65,12,262,48 \\
185,213,477,247\end{array}$ & $\begin{array}{l}55,130,105,198,1028,155,194,114,155,105 \\
124,114,32,288,130\end{array}$ & $2.0369 \mathrm{e}+14$ \\
\hline
\end{tabular}

Table 3. Experimental results in the southern region 


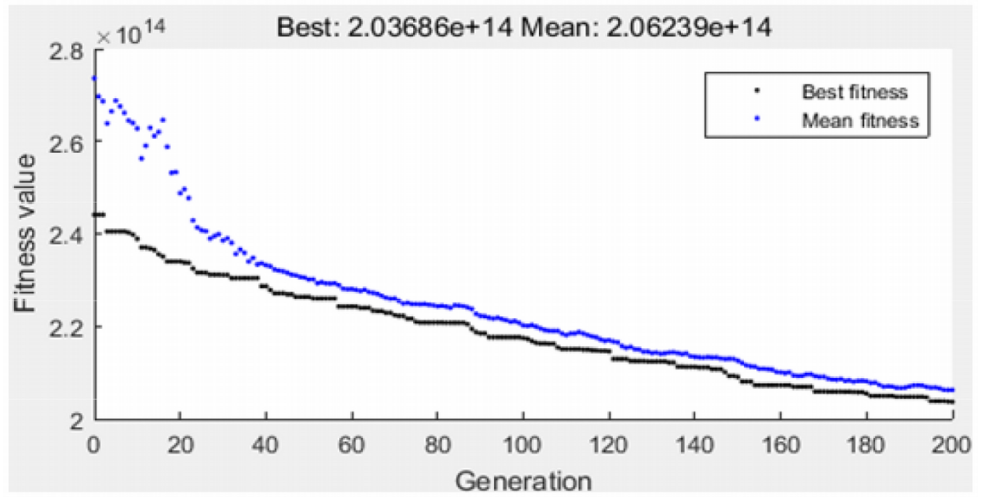

Figure 6. Convergence curve of genetic algorithm in southern, $r=1, \alpha=15$

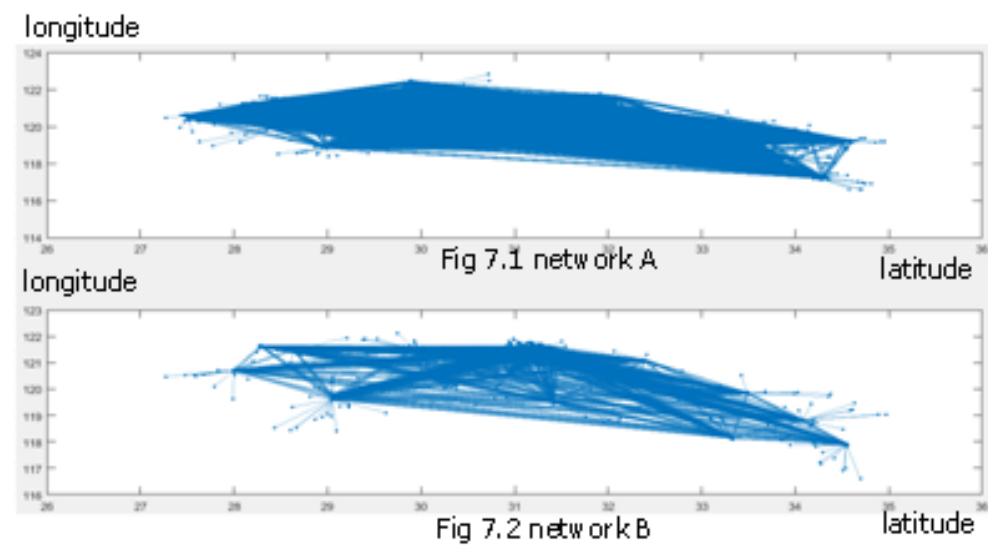

Hub of network $A$ and $B ; O$ spoke of network $A$ and $B$

_ Link of hub and spoke, link of hub and hub in network A and B

Figure 7. The hub and spoke network in the southern region, $r=1, \alpha=15$

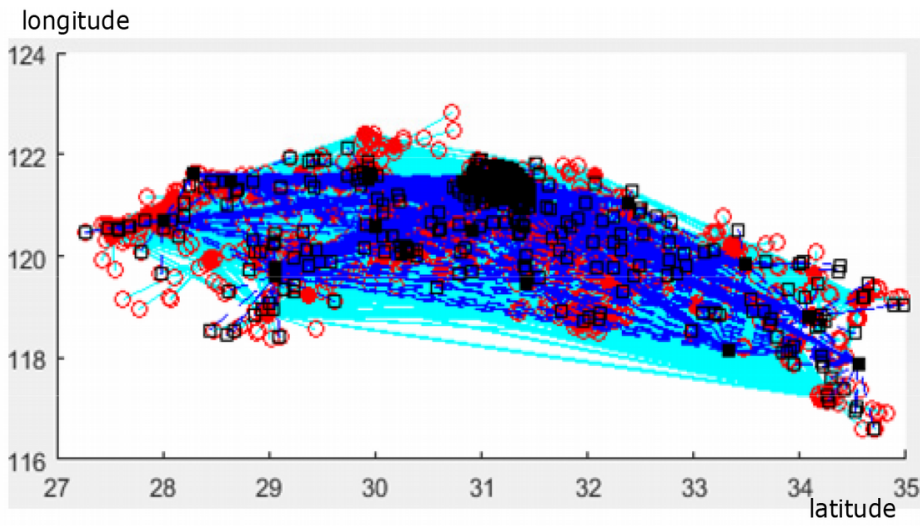

hub of network $A$ spoke of network $A$

The link of hub and spoke, link of hub and hub in network $A$

Hub of network B $\square$ spoke of network B

- " The link of hub and spoke, link of hub and hub of network $B$

Figure 8. Two overlapping hub- and-spoke network of A and B in the southern region, $r=1, \alpha=15$ 
In the process to solve the cargo shunting problem of network A and network B of genetic algorithm, we set the calculation experimental conditions, controlled some parameters in the experiment, and then observed the influence of the change of one parameter on the results, and explained the calculation results based on the influence of the parameters. Parameter sensitivity analysis shows that we can adjust the amount of cargo shunted between the two networks to optimize the total cost according to the different cargo flow sizes in the network, and the transshipment cost between the networks. The change rules of the results obtained from different geographical locations are consistent, and the surface algorithm design is applicable to the different distribution regions of the logistics network.

\section{Conclusion}

The market competition among third-party logistics enterprises is mainly reflected in the competition of logistics network service ability and cost. Logistics companies have to adopt measures to regulate the flow of goods to meet customer demand and reduce costs and a cargo shunt approach that requires cooperation between two logistics companies is discussed. Sample analysis shows that the amount of cargo shunted between the two networks, cargo flow sizes in the network, and the transshipment cost between the networks can affect the total cost and parameters that control traffic and transport costs between networks have an impact on hub flow shunting and cooperation hub between these networks. The calculation example shows the cooperation method of the two networks in two regions of China, the change rules of the results are suitable in different geographical locations.

In the coding of Multilayer genetic algorithm, chromosomes are divided into two segments, each of which carries the information of a hub-and-spoke network. On the one hand, the existing algorithm theory is applied, and on the other hand, some innovations are made in solving new problems. Compared with the study on the internal structure of the hub-and-spoke network, this manuscript pays more attention to the study on the connection and cooperation between the hub-and-spoke networks, and combines the independent hub-and-spoke network to a certain extent to form a new integrated logistics network. The subject provides an idea that the original independent hub-and-spoke network of logistics enterprises can cooperate with each other to build a large logistics network and a comprehensive logistics network, and realize the coordination among regions, lines and nodes, and various modes of transportation.

\section{Declaration of Conflicting Interests}

The authors declared no potential conflicts of interest with respect to the research, authorship, and/or publication of this article.

\section{Funding}

This work is partially supported by the Ministry of Railway Scientific Research Fund (Z2015-C001),the Shanghai Committee of Science and Technology, China,(15dz1100900), the Special project of Shanghai Scientific Research Program,(17DZ2280200).

\section{References}

Alibeyg, A., Contreras, I., \& Fernández, E. (2016). Hub network design problems with profits. Transportation Research Part E: Logistics and Transportation Review, 96, 40-59. https://doi.org/10.1016/j.tre.2016.09.008

Alumur, S.A., Nickel, S., Rohrbeck, B., \& Saldanha-da-Gama, F. (2018). Modeling congestion and service time in hub location problems. Applied Mathematical Modelling, 55, 13-32. https://doi.org/10.1016/j.apm.2017.10.033

Chu, Z., Feng, B., \& Lai, F. (2018). Logistics service innovation by third party logistics providers in China: Aligning guanxi and organizational structure. Transportation Research Part E: Logistics and Transportation Review, 118, 291-307. https://doi.org/10.1016/j.tre.2018.08.007

Cruijssen, F., Cools, M., \& Dullaert, W. (2007). Horizontal cooperation in logistics: Opportunities and impediments. Transportation Research Part E: Logistics and Transportation Review, 43(2), 129-142.

https://doi.org/10.1016/j.tre.2005.09.007 
Cui, L., \& Hertz, S. (2011). Networks and capabilities as characteristics of logistics firms. Industrial Marketing Management, 40(6), 1004-1011. https://doi.org/10.1016/j.indmarman.2011.06.039

Defryn, C., \& Sörensen, K. (2018). Multi-objective optimisation models for the travelling salesman problem with horizontal cooperation. European Journal of Operational Research, 267(3), 891-903.

https://doi.org/10.1016/j.ejor.2017.12.028

Delavar, M.R., Hajiaghaei-Keshteli, M., \& Molla-Alizadeh-Zavardehi, S. (2010). Genetic algorithms for coordinated scheduling of production and air transportation. Expert Systems with Applications, 37, 8255-8266. https://doi.org/10.1016/j.eswa.2010.05.060

Gen, M., Lin, L., Yun, Y., \& Inoue, H. (2018). Recent advances in hybrid priority-based genetic algorithms for logistics and SCM network design. Computers \& Industrial Engineering, 125, 394-412. https://doi.org/10.1016/j.cie.2018.08.025

Hafezalkotob, A. (2017). Competition, cooperation, and coopetition of green supply chains under regulations on energy saving levels. Transportation Research Part E, 97, 228-250. https://doi.org/10.1016/j.tre.2016.11.004

Huang, J., \& Wang, Q. (2009). Robust Optimization of Hub-and-Spoke Airline Network Design Based on Multi-Objective Genetic Algorithm. Journal of Transportation Systems Engineering and Information Technology, 9(3), 8692. https://doi.org/10.1016/S1570-6672(08)60066-5

Ilić, A., Urošević, D., Brimberg, J., \& Mladenović, N. (2010). A general variable neighborhood search for solving the uncapacitated single allocation p-hub median problem. European Journal of Operational Research, 206(2), 289-300. https://doi.org/10.1016/j.ejor.2010.02.022

Ishfaq, R., \& Sox, C.R. (2012). Design of intermodal logistics networks with hub delays. European Journal of Operational Research, 220(3), 629-641. https://doi.org/10.1016/j.ejor.2012.03.010

Jiang, C., Tiziana, D’A, \& Wan, Y. (2017). Air-rail cooperation Partnership level, market structure and welfare implications. Transportation Research Part B, 104, 461-482. https://doi.org/10.1016/j.trb.2017.01.006

Karimi, H., \& Setak, M. (2018). A bi-objective incomplete hub location-routing problem with flow shipment scheduling. Applied Mathematical Modelling, 57, 406-431. https://doi.org/10.1016/j.apm.2018.01.012

Kartal, Z., Hasgul, S., \& Ernst, A.T. (2017). Single allocation p -hub median location and routing problem with simultaneous pick-up and delivery. Transportation Research Part E: Logistics and Transportation Review, 108, 141-159. https://doi.org/10.1016/j.tre.2017.10.004

Kian, R., \& Kargar, K. (2016). Comparison of the formulations for a hub-and-spoke network design problem under congestion. Computers \& Industrial Engineering, 101, 504-512. https://doi.org/10.1016/j.cie.2016.09.019

Li, M., Zhang, Q., \& Deng, Y. (2018). Evidential identification of influential nodes in network of networks. Chaos, Solitons \& Fractals,17, 283-296. https://doi.org/10.1016/j.chaos.2018.04.033

Liu, W., Wang, D., Shen, X., Yan, X., \& Wei, W. (2018). The impacts of distributional and peer-induced fairness concerns on the decision-making of order allocation in logistics service supply chain. Transportation Research Part E: Logistics and Transportation Review, 116, 102-122. https://doi.org/10.1016/j.tre.2018.05.006

Long, Q. (2016). A flow-based three-dimensional collaborative decision-making model for supply-chain networks. Knowledge-Based Systems, 97, 101-110. https://doi.org/10.1016/j.knosys.2016.01.012

Long, Q. (2017). A framework for data-driven computational experiments of inter-organizational collaborations in supply chain networks. Information Sciences, 399, 43-63. https://doi.org/10.1016/j.ins.2017.03.008

Martins, E., Contreras, I., \& Cordeau, J.F. (2015). Exact and heuristic algorithms for the design of hub networks with multiple lines. European Journal of Operational Research, 246(1), 186-198.

https://doi.org/10.1016/j.ejor.2015.04.017

Mesa-Arango, R., \& Ukkusuri, S.V. (2015). Demand clustering in freight logistics networks. Transportation Research Part E: Logistics and Transportation Review, 81, 36-51. https://doi.org/10.1016/j.tre.2015.06.002 
Moradinasab, N., Amin-Naseri, M.R., Behbahani, T.J., Jafarzadeh, H. (2018). Competition and cooperation between supply chains in multiobjective petroleum green supply chain: A game theoretic approach. Journal of Cleaner Production, 170, 818-841. https://doi.org/10.1016/j.jclepro.2017.08.114

Niknejad, A., \& Petrovic, D. (2014). Optimisation of integrated reverse logistics networks with different product recovery routes. European Journal of Operational Research, 238(1), 143-154. https://doi.org/10.1016/j.ejor.2014.03.034

O’Kelly, M.E. (2008). Routing Traffic at Hub Facilities. Networks and Spatial Economics, 10(2), 173-191. https://doi.org/10.1007/s11067-008-9061-z

Ping, L. (2014). An Empirical Analysis of China's Trade Effect under the ramework of CAFTA (master). Yunan University of Finance and Economics, Yunan.

Shekhtman, L.M., Danzinger, M.M., Vaknin, D., \& Havlin, S. (2018). Robustness of spatial networks and networks of networks (Robustesse des réseaux spatiaux et des réseaux de réseaux). Comptes Rendus Physique, 19(4), $233-243$. https://doi.org/10.1016/j.crhy.2018.09.005

Shi, F., Wang, H., \& Yu, L. (2011). 30 case studies of MATLAB intelligent algorithm. Beijing: Beihang University Press.

Wang, Y., Ma, X., Liu, M., Gong, K., Liu, Y., Xu, M. et al. (2017). Cooperation and profit allocation in two-echelon logistics joint distribution network optimization. Applied Soft Computing, 56, 143-157.

https://doi.org/10.1016/j.asoc.2017.02.025

Wang, Y., Peng, S., Xu, C., Assogba, K., Wang, H., Xu, M. et al. (2018). Two-echelon logistics delivery and pickup network optimization based on integrated cooperation and transportation fleet sharing. Expert Systems with Applications, 113, 44-65. https://doi.org/10.1016/j.eswa.2018.06.037

Wang, Y., Zhang, J., Assogba, K., Liu, Y., Xu, M., \& Wang, Y. (2018). Collaboration and transportation resource sharing in multiple centers vehicle routing optimization with delivery and pickup. Knowledge-Based Systems, 160, 296-310. https://doi.org/10.1016/j.knosys.2018.07.024

Woo, Y.B. \& Kim, B.S. (2019). A genetic algorithm-based matheuristic for hydrogen supply chain network problem with two transportation modes and replenishment cycles. Computers \& Industrial Engineering, 127, 981-997. https://doi.org/10.1016/j.cie.2018.11.027

Yuan, Y., \& Yu, J. (2018). Locating transit hubs in a multi-modal transportation network: A cluster-based optimization approach. Transportation Research Part E: Logistics and Transportation Review, 114, 85-103. https://doi.org/10.1016/j.tre.2018.03.008

Zhang, H., Deng, Y., Chan, F. T. S., \& Zhang, X. (2013). A modified multi-criterion optimization genetic algorithmfor order distribution in collaborative supply chain. Applied Mathematical Modelling, 37, 7855-7864. https://doi.org/10.1016/j.apm.2013.05.021

Journal of Industrial Engineering and Management, 2019 (www.jiem.org)

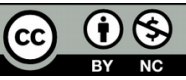

Article's contents are provided on an Attribution-Non Commercial 4.0 Creative commons International License. Readers are allowed to copy, distribute and communicate article's contents, provided the author's and Journal of Industrial Engineering and Management's names are included. It must not be used for commercial purposes. To see the complete license contents, please visit https://creativecommons.org/licenses/by-nc/4.0/. 